\title{
Estado Nutricional y Desempeño Físico de una muestra de escolares de 14 y 15 años de la ciudad de Chillán, Chile
}

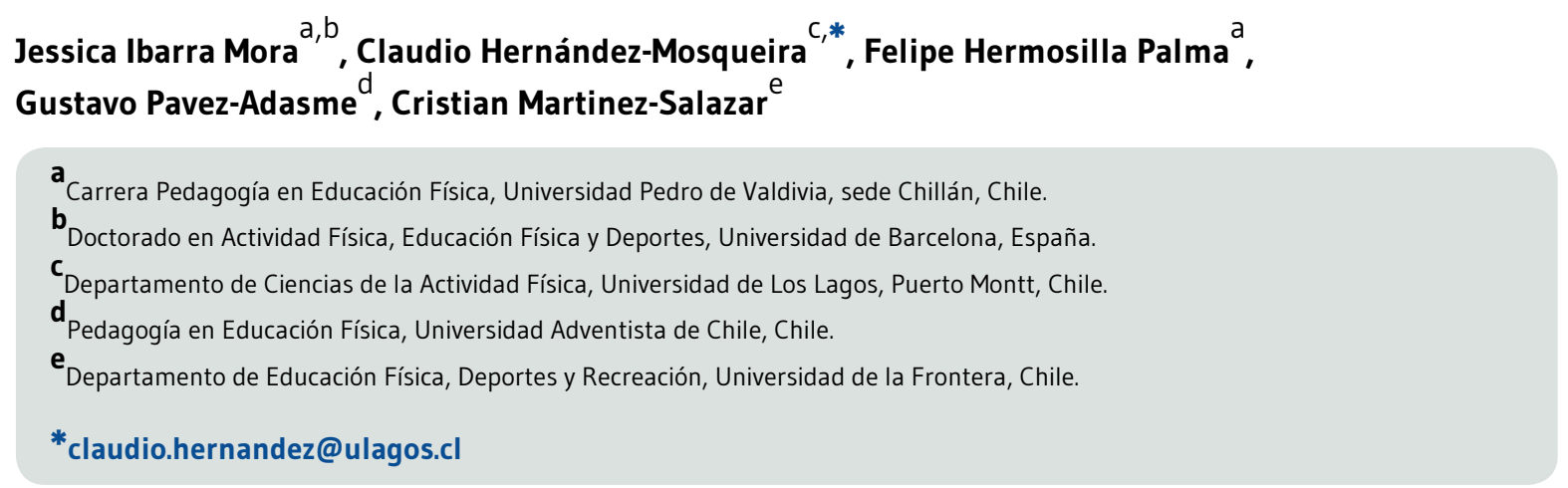

Recibido el 3 de marzo de 2017; aceptado el 15 de junio de 2017; publicado el 25 de septiembre de 2017.

\section{PALABRAS CLAVE}

Aptitud Física;

Estado Nutricional;

Estudiantes.
Estado Nutricional y Desempeño Físico de una muestra de escolares de 14 y 15 años de la ciudad de Chillán, Chile

\section{RESUMEN}

Introducción: La obesidad infantil es un problema de elevada magnitud y con graves repercusiones en el estado de salud, lo que plantea la necesidad de tomar medidas orientadas a estimular o mejorar, tanto el aporte nutricional como la condición física. El objetivo de este estudio fue comparar el desempeño físico de una muestra de estudiantes según el estado nutricional en estudiantes de 14 y 15 años de la ciudad de Chillán.

Material y Métodos: Estudio observacional transversal descriptivo llevado a cabo con 245 estudiantes del instituto superior de Comercio de la ciudad de Chillán, octava región de Chile. Se recogió información del peso y talla con el que se obtuvo el índice de masa corporal y la circunferencia cintura. Además se recogió información sobre el desempeño físico como Course-Navette, abdominales, flexiones de brazo, salto y flexibilidad a través de la batería utilizada por la Agencia de Calidad de la Educación en Chile (SIMCE) de Educación Física.

Resultados: Un $18,8 \%$ y un $14,3 \%$ de los estudiantes presenta sobrepeso y obesidad respectivamente. Los niños que presentan sobrepeso y obesidad tienen un peor rendimiento físico con un menor número de repeticiones abdominales y flexiones de brazo, un menor salto y un menor tiempo en segundos en Course-Navette. En flexibilidad no se encontraron diferencias según es estado nutricional.

Conclusiones: Los estudiantes con sobrepeso y obesidad presentan un peor rendimiento físico. De ahí la necesidad de tomar medidas orientadas a estimular o mejorar la capacidad física para revertir estas tendencias adversas para su salud. 


\section{KEYWORDS}

Physical Fitness;

Nutritional Status;

Students.
Nutritional Status and Physical Performance of a sample of 14 and 15 year old schoolchildren from the city of Chillán, Chile

\section{ABSTRACT}

Introduction: Childhood obesity is a huge problem with serious consequences on the state of health, raising the need to take measures to stimulate or improve both the nutritional intake and physical condition. The aim of this study was to assess nutritional status according physical performance in a sample of students aged 14 and 15 years of the city of Chillan.

Material and Methods: A descriptive cross-sectional study carried out with 245 students from the upper institute of Commerce of the city of Chillán, eighth region of Chile. Weight and height were collected to obtain the body mass index and waist circumference. Moreover, physical performance were collected such as Course-Navette, abdominals, arm push up, jumping and flexibility using the battery used by the Education Quality Agency in Chile (SIMCE) of Physical Education.

Results: $18.8 \%$ and $14.3 \%$ of the students were overweight and obese, respectively. The overweight and obese students have a poorer physical performance with fewer abdominaland arm push ups, a lower jump and a shorter time in seconds on the Course-Navette. In flexibility score no differences were found according to nutritional status.

Conclusions: Overweight and obesity students had worse physical performance. Hence the need to take measures aimed at stimulating or improving physical capacity to reverse these adverse trends for their health.

\section{CITA}

Ibarra Mora J, Hernández-Mosqueira C, Hermosilla Palma F, Pavez-Adasme G, Martinez-Salazar C. Estado Nutricional y Desempeño Físico de una muestra de escolares de 14 y 15 años de la ciudad de Chillán, Chile. Rev Esp Nutr Hum Diet. 2017; 21(3): 248-55. doi: 10.14306/renhyd.21.3.363

\section{INTRODUCCIÓN}

El sedentarismo es un problema de salud en Chile desde hace más de 20 años. Los efectos negativos del sedentarismo en la salud es también una fuente de preocupación, ya que se ha demostrado que los sujetos que presenten niveles altos de adiposidad y de sedentarismo tienen un mayor riesgo cardiovascular ${ }^{1}$. De acuerdo al estudio entre 1988 y 2010 de Ladabaum², el estilo de vida sedentario es la principal causa del aumento en los índices de obesidad en la población adulta de Estados Unidos. En el caso de Latinoamérica se estima que entre 42,5 y 51,8 millones de niños y adolescentes (0-18 años) presentan sobrepeso u obesidad, lo que representa el $20-25 \%$ del total población de todos los niños y adolescentes de la región ${ }^{3}$. Una revisión sistemática con publicaciones entre los años 2008 y 2013, para estimar la prevalencia de sobrepeso y obesidad en niños de 0-19 años en América Latina, observó que 3,8 millones de niños menores de 5 años, 22,2-25,9 millones de niños en edad escolar y 16,5-21,1 millones de adolescentes presentaban sobrepeso u obesidad. En general, entre 42,5 y 51,8 millones de niños de 0 a 19 años de edad fueron afectados, es decir, alrededor del 20-25\% de la población4. En Chile, el 80,1\% de la población mayor de 18 años se declara sedentaria y esta falta de actividad física está relacionada directamente con enfermedades crónicas no transmisibles, además de ser un factor de riesgo para la diabetes y la hipertensión, entre otras dolencias del sistema cardio o cerebrovascular ${ }^{5}$. Por otra parte, la Encuesta Nacional de Salud ${ }^{6}$, reveló cifras alarmantes de obesidad, hipertensión arterial, dislipidemias y sedentarismo en la población mayor de 17 años. Esto cobra importancia, debido a que la obesidad infantil si se manifiesta o persiste en la segunda década de vida, es muy probable que en la edad adulta se sufra de obesidad, sobre todo con enfermedades cardiovasculares, tal como lo plantean las investigaciones ${ }^{7,8}$. En esta perspectiva, diversos 
Estado Nutricional y Desempeño Físico de una muestra de escolares de 14 y 15 años de la ciudad de Chillán, Chile

estudios plantean que la mejor manera de prevenir la obesidad infantil y combatir los diversos problemas de salud asociados a ésta es la práctica regular de actividad física9,10. El ejercicio físico, practicado de manera apropiada, es quizás la mejor herramienta hoy disponible para fomentar la salud y el bienestar de la persona. De manera directa y específica, el ejercicio físico mantiene y mejora la función músculo-esquelética, osteo-articular, cardio-circulatoria, respiratoria, endocrino-metabólica, inmunológica y psiconeurológica ${ }^{11,12}$. En el ámbito educativo, la condición física se vincula fundamentalmente con la salud, y considera aquellos componentes que se ven afectados favorable o negativamente por el nivel habitual de actividad física, que están relacionados directamente con un estado de vida saludable ${ }^{13}$. Por ello la escuela se reconoce como una forma efectiva de intervención para mejorar y proteger la salud de la comunidad escolar, y que precisa de la colaboración intersectorial entre educación y salud ${ }^{14}$. En este sentido, en Chile desde el año 2010 se utiliza a nivel nacional el SIMCE de Educación Física, que es una batería de test para evaluar el rendimiento físico de escolares de 13 y 14 años. El último SIMCE de Educación Física ${ }^{15}$, señala que un $44 \%$ de los estudiantes chilenos de octavo año básico se encuentra con sobrepeso u obesidad, presentando además bajos niveles de condición física y capacidad cardiorrespiratoria. Este es un factor fundamental a analizar, ya que se ha demostrado una directa relación entre un bajo nivel cardiorrespiratorio con diversas patologías, en especial con enfermedades cardiometabólicas, como lo son las enfermedades cardiacas, diabetes mellitus tipo Il y síndrome metabólico, entre otras ${ }^{16}$. De acuerdo a lo planteado, el objetivo del presente estudio es valorar la condición nutricional y desempeño físico de una muestra de escolares de 14 y 15 años de la ciudad de Chillán, Chile.

\section{material y métodos}

El presente estudio observacional transversal descriptivo, se llevó a cabo en la ciudad de Chillán, octava región de Chile, con una población de 360 estudiantes en edad escolar de 14 y 15 años, pertenecientes al Instituto superior de Comercio (INSUCO). Se realizó un muestreo de tipo no probabilístico, con selección de manera no aleatoria por conveniencia, y se consideraron los siguientes criterios de inclusión: i) tener una condición de salud que le permitiera realizar ejercicio físico; ii) tener rendimiento en el Test de Cafra inferior a 160 latidos por minuto; iii) haber asistido a clases el día de la aplicación de las pruebas. Se excluyeron a los estudiantes que no cumplieran con estos requisitos, quedando la muestra en 245 . De ellos 120 son hombres y 125 mujeres que representa el $25 \%$ de los estudiantes del colegio.

La participación era voluntaria, todos los participantes fueron orientados en cuanto a los procedimientos a ser realizados para la aplicación de los test de condición física y recibieron información acerca de los fines, objetivos y métodos del estudio antes del inicio de las evaluaciones. Cada apoderado o tutor debió firmar un consentimiento informado para que su hijo participara en el estudio, de esta forma se respetó las normas de Helsinki que se refieren a la investigación con seres humanos.

El índice de masa corporal (IMC; $\mathrm{kg} / \mathrm{m}^{2}$ ) se calculó a partir del peso y la talla de los alumnos. La medición del peso y talla se hizo de acuerdo a las normas estandarizadas de la Sociedad Internacional para el Avance de la Cineantropometría $(\mathrm{ISAK})^{17}$. Para medir el peso y la talla se utilizó un estadiómetro marca Seca ${ }^{\circledR}$, graduado en milímetros y gramos. Con ello se determinó el grado de obesidad, determinando el estatus de peso corporal de los participantes y clasificándolos en bajo peso, normopeso, sobrepeso y obesidad, de acuerdo a la norma técnica de evaluación nutricional del niño de 6 a 18 años $^{18}$. La circunferencia de cintura se midió de acuerdo a las normas ISAK ${ }^{17}$, empleando una cinta métrica marca Cescorf (precisión de $1 \mathrm{~mm}$ ). Los test que permitieron evaluar el rendimiento físico fueron los utilizados por la Agencia de Calidad de la Educación ${ }^{19}{ }^{19}$ en el orden descrito, que consisten en los siguientes:

a) El test de Cafra: tiene como objetivo detectar alumnos que tengan riesgos cardiovasculares para eximirlos de rendir el test de Course-Navette. Consiste en que el estudiante debe caminar manteniendo una velocidad constante de $6 \mathrm{~km} / \mathrm{h}$ durante 3 minutos sobre un pentágono en el que cada lado mide 10 metros, al ritmo de un estímulo sonoro, al término de la prueba se controla y anota la frecuencia cardiaca. Si al finalizar la prueba el alumno presenta una frecuencia cardiaca igual o mayor a 160 pulsaciones por minuto, no debe rendir el test de CourseNavette. Los escolares que superaron estas pulsaciones fueron descartados del estudio.

b) Test de abdominales en 30 segundos: su objetivo es evaluar la fuerza de la musculatura flexora del tronco. El estudiante debe realizar el mayor número de repeticiones posibles durante 30 segundos. Fueron utilizadas colchonetas marca Bronson y cronómetro Casio ${ }^{\circledR} \mathrm{HS}-80$ TW-1EF.

c) Salto largo a pies juntos: su objetivo es evaluar la fuerza de las extremidades inferiores, su desarrollo consiste en que el estudiante debe realizar un salto longitudinal y lograr la mayor distancia posible de desplazamiento 
horizontal. Para este test se utilizó una superficie lisa, tiza y una huincha (cinta).

d) Flexo-extensión de codo: el objetivo de este test es evaluar la fuerza extensora de la musculatura del codo. El estudiante se debe ubicar en posición paralela a una superficie plana (suelo). Los hombres deben usar como puntos de apoyo las manos y los pies, y las mujeres las manos y las rodillas. Se deben realizar la mayor cantidad de flexo-extensiones de codo, en 30 segundos.

e) Flexión de tronco adelante: esta prueba se realiza para medir la flexibilidad de la espalda baja y de los músculos que se encuentran en la región posterior del muslo. Para evaluar la flexibilidad se utilizó un banco marca Terrazul ${ }^{\circledR}$ modelo Wells Physical de estructura MDF de $18 \mathrm{~mm}$; alto: $31 \mathrm{~cm}$; longitud medida: 64,5cm; largo: $40 \mathrm{~cm}$; espesor: 2cm; peso: $3 \mathrm{~kg}$.

f) Test de Course-Navette: tiene como objetivo medir la resistencia aeróbica máxima, es decir, la capacidad que tiene el cuerpo para suministrar el oxígeno necesario a los músculos durante un esfuerzo máximo, Su desarrollo consiste en que el estudiante se debe desplazar recorriendo 20m, aumentando progresivamente la velocidad e intensidad de su desplazamiento: al inicio debe ir caminando, luego trotando y al final corriendo. Este desplazamiento se realiza durante 15 minutos, al ritmo de un pulso sonoro que acelera progresivamente. Para evaluar, se utilizó un gimnasio donde se delimitó la distancia a ser recorrida por conos, un cronómetro Casio ${ }^{\circledR} \mathrm{HS}-80$ TW-1EF función árbitro con una exactitud de una milésima de segundo.

Para garantizar el nivel de habilidad de los evaluadores, fue realizada con anterioridad una prueba piloto en el propio grupo. La recolección de los datos fue supervisada por profesores de educación física con experiencia en este tipo de evaluación.

Para el análisis estadístico, se utilizó el software SPSS 20.0 (SPSS Inc., IL, EE.UU.). La distribución de normalidad fue comprobada con el test de Kolmogorov-Smirnov. Se calculó la media (desviación estándar) y porcentajes para describir la valoración nutricional y de condición física de la muestra. Para la comparación de medias de acuerdo al sexo de los participantes se utilizó el test de Student, y para la comparación del estado nutricional y desempeño físico se utilizó el análisis de varianza (ANOVA) de un factor entre sujetos con post hoc de Tukey. Para todas las pruebas estadísticas se fijó un nivel de significación de $p<0,05$.

\section{RESULTADOS}

En la Tabla 1 se presentan las características de edad, estado nutricional y rendimiento físico según hombres y mujeres. Se observan diferencias significativas en el peso, estatura e

Tabla 1. Características de edad, estado nutricional y rendimiento físico de la muestra estudiada de acuerdo al sexo.

\begin{tabular}{l|c|c|c} 
& $\begin{array}{c}\text { Mujeres } \\
(\mathbf{n = 1 2 5 )}\end{array}$ & $\begin{array}{c}\text { Hombres } \\
(\mathbf{n}=120)\end{array}$ & Valor p \\
\hline Edad (años) & $14,7(0,5)$ & $14,6(0,5)$ & 0,70 \\
Peso (kg) & $57,4(11,3)$ & $62,0(12,8)$ & 0,03 \\
Estatura (cm) & $158,9(0,1)$ & $168,5(0,1)$ & $<0,001$ \\
IMC (kg/m²) & $22,9(4,0)$ & $21,8(3,9)$ & 0,02 \\
Normal (\%) & $79,0(63,2)$ & $85,0(70,8)$ & \\
Sobrepeso (\%) & $25,0(20,0)$ & $21,0(17,5)$ & \\
Obesidad (\%) & $21,0(16,8)$ & $14,0(11,7)$ & \\
Circunferencia de cintura (cm) & $75,5(9,3)$ & $77,5(10,4)$ & 0,11 \\
Course-Navette (min) & $2,9(1,3)$ & $4,6(2,2)$ & $<0,001$ \\
Abdominales (repeticiones) & $14,5(8,9)$ & $20,8(7,0)$ & $<0,001$ \\
Flexiones de Brazo (repeticiones) & $14,5(7,6)$ & $18,1(10,2)$ & 0,01 \\
Salto (cm) & $126,7(0,2)$ & $173,1(0,3)$ & 0,03 \\
Flexibilidad (cm) & $24,9(9,4)$ & $21,1(9,8)$ & 0,03 \\
\hline
\end{tabular}

Los resultados se expresan en media ( \pm desviación estándar). Prueba T Student para la diferencia de medias, diferencias significativas ( $p<0,05)$. IMC: Índice de Masa Corporal. 
IMC entre hombres y mujeres. Los hombres presentaban un punto menos en el IMC que las mujeres. En el rendimiento físico se observó diferencias significativas en todos los parámetros, siendo ligeramente superiores en los hombres que en las mujeres, con excepción del de flexibilidad, en el que las mujeres presentaban tres puntos más en media que los hombres $(p<0,03)$.

En el presente estudio el 66,9\% de la muestra total presentó normopeso (164 personas), el 18,8\% sobrepeso (46 personas) y un $14,3 \%$ obesidad (35 personas). En la Tabla 2, se presenta el rendimiento físico según el estado nutricional de los estudiantes. Los niños que presentan sobrepeso y obesidad tienen un peor rendimiento físico. En general los niños con sobrepeso y obesidad presentaban menos repeticiones abdominales y flexiones de brazo, un menor salto y un menor tiempo en segundos en Course-Navette $(p<0,05)$. En flexibilidad no se encontraron diferencias según el estado nutricional.

\section{Discusión}

El propósito del estudio fue valorar el estado nutricional y desempeño físico de una muestra de estudiantes de 14 y 15 años de la ciudad de Chillán, Chile. Diversos estudios han mostrado que en niños y adolescentes, al presentar mejor estado nutricional, es mayor el rendimiento físico en los test de tipo aeróbico ${ }^{20-22}$. En cuanto al IMC, este es considerado como el mejor indicador antropométrico para diagnosticar el sobrepeso y la obesidad en niños entre los 2 y los 18 años ${ }^{20,23}$. El promedio de IMC en los estudiantes del INSUCO de Chillán fue de 22,9 y $21,8 \mathrm{~kg} / \mathrm{m}^{2}$ en mujeres y hombres respectivamente, diferente a lo reportado por Khan y Cols. ${ }^{24}$ cuyo estudio se realizó con escolares canadienses de 6 a 17 años y en donde los hombres poseían valores superiores $\left(20,6 \mathrm{~kg} / \mathrm{m}^{2}\right)$ y $20,2 \mathrm{~kg} / \mathrm{m}^{2}$ las mujeres. Pero al ser comparados con estudiantes Chilenos de Padre las Casas resultan similares, con un 22,1 y $21,3 \mathrm{~kg} / \mathrm{m}^{2}$ en mujeres y hombres respectivamente ${ }^{16}$. Respecto del estado nutricional, nuestro estudio indica que la prevalencia de sobrepeso y obesidad están por debajo de la media, con un $18,8 \%$ de sobrepeso y un $14,3 \%$, al ser comparados con: i) el estudio de Caamaño y Cols. ${ }^{25}$ realizado con escolares de 11 a 16 años de la ciudad de Temuco, en el que se situaba el porcentaje de niños con sobrepeso en un 30,9\% y de obesidad en 17,3\%; ii) el de Hernández-Mosqueira y Cols. ${ }^{26}$ realizado en la ciudad de Chillán, donde se reporta un $39,4 \%$ de sobrepeso y un $28,8 \%$ de obesidad; y iii) los datos reportados por el Ministerio de Educación de Chile en una muestra nacional de escolares, donde el 40,2\% de ellos presentaban alteraciones del peso ${ }^{19}$. Sin embargo, llama la atención que al ser contrastados con estudios internacionales los resultados se encuentran sobre la media de sobrepeso y obesidad, como el de Figueroa y Cols. ${ }^{27}$ en Brasil, Ganie y Cols. ${ }^{28}$ en India, Jakšić y Cols. ${ }^{29}$ en Montenegro, que presentaron sólo un $12,3 \%$ y $9,2 \% ; 4,6 \%$ y $3,3 \% ; 18,9 \%$ y $12,1 \%$ de sobrepeso y obesidad respectivamente. Al analizar los resultados por sexo, se puede apreciar que el $24,8 \%$ de las mujeres tiene sobrepeso y el $12,8 \%$ tiene obesidad, porcentajes similares al estudio de HernándezCordero y Cols. ${ }^{30}$, con $23,7 \%$ y $12,1 \%$ respectivamente. Lo mismo sucede con los porcentajes correspondientes a los hombres, quienes presentan un $16,7 \%$ sobrepeso y $12,5 \%$ de obesidad respecto al citado estudio con $19,6 \%$ y $14,5 \%$. En la obesidad infantil esto tiene especial trascendencia, ya que la obesidad y la obesidad abdominal se asocian de forma independiente con morbilidad y mortalidad ${ }^{2}$. Algunos estudios realizados en Chile han mostrado que los escolares obesos presentan un incremento significativo en las medidas antropométricas, necesitan mayor tiempo para recorrer $400 \mathrm{~m}$, poseen niveles aumentados de glucosa basal y un aumento del riesgo de cardiometabólico ${ }^{12,20,23}$.

Tabla 2. Características de rendimiento físico de acuerdo al estado nutricional.

\begin{tabular}{l:c:c:c:c} 
Características & Normal & Sobrepeso & Obesidad & Valor $p$ \\
\hdashline Course-Navette (min) & $4,2(2,0)$ & $3,2(1,7)$ & $2,2(1,3)$ & $<0,001$ \\
Abdominales (repeticiones) & $19,4(7,9)$ & $14,2(9,1)$ & $13,7(8,9)$ & $<0,001$ \\
Flexiones de Brazo (repeticiones) & $18,2(9,0)$ & $14,1(8,3)$ & $10,1(7,7)$ & $<0,001$ \\
Salto (cm) & $155,9(0,3)$ & $145,1(0,3)$ & $124,9(0,3)$ & $<0,001$ \\
Flexibilidad (cm) & $23,5(10,3)$ & $23,1(8,3)$ & $20,6(9,0)$ & 0,285
\end{tabular}

Los resultados se expresan en media ( $₫$ desviación estándar). Prueba ANOVA para la diferencia de medias, diferencias significativas ( $p<0,05)$. IMC: Índice de Masa Corporal. 
Estado Nutricional y Desempeño Físico de una muestra de escolares de 14 y 15 años de la ciudad de Chillán, Chile

Respecto del desempeño físico en la presente investigación, los estudiantes con sobrepeso u obesidad presentaron rendimientos significativamente menores en flexibilidad, abdominales, flexiones de brazo, salto, Course-Navette, siendo estos resultados similares a los reportados por Caamaño y Cols. $^{25}$. El alto grado de sobrepeso detectado en esta investigación sugiere que la muestra sería sedentaria ${ }^{31}$, concordando con el estudio de Vásquez y Cols., donde los escolares chilenos presentan un patrón de actividad predominantemente sedentario, con más de 10 horas de actividades de bajo costo energético ${ }^{32}$. Al analizar los resultados por sexo, se puede apreciar que los hombres tuvieron un rendimiento significativamente mayor en abdominales, flexiones de brazo, salto, Course-Navette, similar a lo descrito por Ranson y Cols. ${ }^{33}$, quienes reportaron que los hombres superaron significativamente a las mujeres en el test de $10 \times 5 \mathrm{~m}$, en el test de $20 \mathrm{~m}$, en el salto de longitud y en la fuerza de prensión manual. Las mujeres no alcanzan el nivel aceptable en ninguna de las pruebas evaluadas, de acuerdo a los valores de referencia establecidos en el Informe Nacional de Educación Física (SIMCE, 2015) ${ }^{23}$, excepto en abdominales, donde las estudiantes con bajo peso y normopeso, alcanzan el promedio las 16 repeticiones, considerándose como aceptable. Al comparar las mujeres normopeso con el estudio de Cárcamo y Cols. ${ }^{34}$ en estudiantes de la Región de Aysén, Chile, se observan resultados bajos en la prueba de flexibilidad y de salto largo a pies juntos, ya que los resultados de nuestro estudio nos indican $24,9 \mathrm{~cm}$ y $126,7 \mathrm{~cm}$ respectivamente frente a $33,6 \mathrm{~cm}$ y $130,5 \mathrm{~cm}$. Sólo en la prueba de flexo-extensiones de codo se presentan mejores resultados, 14,5 repeticiones frente a 12,4 . Esta tendencia no se observa con los hombres normopeso ya que únicamente en la prueba de flexibilidad se obtienen resultados mayores a nuestro estudio, $30,2 \mathrm{~cm}$ frente a $21,1 \mathrm{~cm}$; y en la prueba de flexo-extensiones de brazo y de salto largo a pies juntos son mejores los resultados del presente estudio, 18,1 repeticiones y $173,1 \mathrm{~cm}$ respectivamente frente a 10,9 repeticiones y $166,0 \mathrm{~cm}$. Pero al ser comparados con el Informe Nacional de Educación Física (SIMCE, 2015)23, de la misma forma que las mujeres, los hombres en promedio, no alcanzan en nivel aceptable en ninguna de las pruebas, excepto los estudiantes con normopeso, que alcanzan el promedio aceptable en las pruebas de abdominales, flexo-extensión de codos y salto. Estos resultados pueden ser explicados de acuerdo al nivel de sobrepeso y obesidad detectado, ya que un IMC elevado disminuye negativamente el rendimiento en pruebas de salto ${ }^{35}$. Al igual que en otros estudios, los escolares con una mejor condición física a nivel cardiovascular presentan menores valores de $\mathrm{IMC}^{20,36}$, como sucede en el presente estudio. Es recomendable mantener niveles adecuados de aptitud cardiorrespiratoria, ya que el aumento de un valor de equivalente metabólico (MET) disminuye en un 13 a $15 \%$ el riesgo de mortalidad ${ }^{37}$. Finalmente, los resultados evidencian un bajo nivel de condición física en mujeres y hombres. En aspectos estructurales, se reitera la tendencia del Informe Nacional de Educación Física $(2015)^{23}$, donde un $0 \%$ de los estudiantes de la Región del Bío Bío logró la categoría aceptable en todas las pruebas. Entre las limitaciones de nuestro estudio podemos mencionar que estos resultados pueden ser atribuidos a otras variables que en la presente investigación no fueron estudiadas, tales como el aumento de los ingresos, sedentarismo, la influencia de los medios de comunicación y el marketing de alimentos, ya que son factores que influyen en la condición física de los niños en edad escolar ${ }^{38-40}$. Adicionalmente, los resultados obtenidos deben ser tratados con precaución. Es posible que las puntuaciones obtenidas puedan variar en función de las diferencias entre países o regiones. En Chile se han encontrado diferencias en la actividad física que los escolares realizan en función de la región ${ }^{41}$, por lo que se necesitan futuros estudios con otras poblaciones para evaluar estos aspectos mencionados y aumentar el número de estudiantes a fin de responder a estos interrogantes.

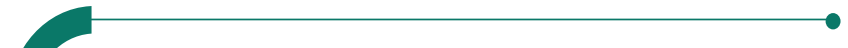 CONCLUSIONES}

Los estudiantes con sobrepeso y obesidad tienen peor rendimiento físico, esto plantea la necesidad de tomar medidas orientadas a estimular o mejorar la capacidad física para revertir estas tendencias adversas para su salud.

\section{AGRADECIMIENTOS}

Agradecemos a las autoridades del INSUCO, Chillán, quienes dieron las facilidades necesarias para poder realizar la investigación, a los niños que participaron, y especialmente al grupo de alumnos, profesores y ayudantes de la carrera de Pedagogía en Educación Física de la Universidad Pedro de Valdivia, sede Chillán.

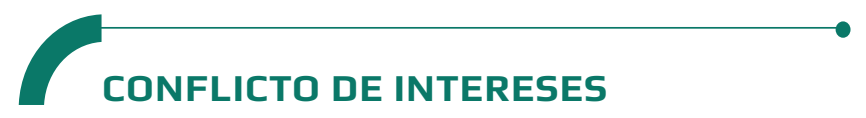

Los autores expresan que no hay conflictos de interés al redactar el manuscrito. 


\section{$\longrightarrow$ REFERENCIAS}

(1) Martínez-Gómez D, Eisenmann JC, Gómez-Martínez S, Veses A, Marcos A, Veiga OL. Sedentarismo, adiposidad y factores de riesgo cardiovascular en adolescentes. Estudio AFINOS. Rev Esp Cardiol. 2010; 63(3): 277-85.

(2) Ladabaum U, Mannalithara A, Myer PA, Singh G. Obesity, abdominal obesity, physical activity, and caloric intake in US adults: 1988 to 2010. Am J Med. 2014; 127(8): 717-727.e12.

(3) Black RE, Victora CG, Walker SP, Bhutta ZA, Christian P, de Onis $M$, et al. Maternal and child undernutrition and overweight in low-income and middle-income countries. Lancet. 2013; 382(9890): 427-51.

(4) Rivera JÁ, de Cossío TG, Pedraza LS, Aburto TC, Sánchez TG, Martorell R. Childhood and adolescent overweight and obesity in Latin America: a systematic review. Lancet Diabetes Endocrinol. 2014; 2(4): 321-32.

(5) Ministerio del Deporte - Gobierno de Chile. Encuesta Nacional de Hábitos de Actividad Física y Deportes 2015 [Internet]. Ministerio del Deporte - Gobierno de Chile. 2016 [citado 15 de septiembre de 2016]. Disponible en: http://www.mindep.cl/ fotos-encuesta-actividad-fisica/

(6) Ministerio de Salud - Gobierno de Chile. Encuesta Nacional de Salud ENS Chile (2009-2010) [Internet]. Santiago de Chile: Ministerio de Salud - Gobierno de Chile; 2011. Disponible en: http://web.minsal.cl/portal/url/item/ bcb03d7bc28b64dfe040010165012d23.pdf

(7) Yeste D, Carrascosa A. Complicaciones metabólicas de la obesidad infantil. An Pediatr. 2011; 75(2): 135.e1-135.e9.

(8) Colomer Revuelta J, Grupo Previnfad. Prevención de la obesidad infantil. Rev Pediatr Aten Primaria. 2005; 7(26): 255-75.

(9) Haugen T, Ommundsen $Y$, Seiler $S$. The relationship between physical activity and physical self-esteem in adolescents: the role of physical fitness indices. Pediatr Exerc Sci. 2013; 25(1): 138-53.

(10) Palou P, Vidal J, Ponseti FJ, Cantallops J, Borras PA. Relaciones entre calidad de vida, actividad física, sedentarismo y fitness cardiorrespiratorio en niños. Rev Psicol Deporte. 2012; 21(2): 393-8.

(11) Castillo MJ. La condición física es un componente importante de la salud para los adultos de hoy y de mañana. Selección. 2007; 16(1): 2-8.

(12) Vásquez F, Díaz E, Lera L, Meza J, Salas I, Rojas P, et al. Impacto del ejercicio de fuerza muscular en la prevención secundaria de la obesidad infantil: intervención al interior del sistema escolar. Nutr Hosp. 2013; 28(2): 347-56.

(13) Lamela M. Valoración de la Condición Física relacionada con la salud en el ámbito educativo [Internet]. CFR Lugo; 2009. Disponible en: http://centros.edu.xunta.es/cfr/lugo/files/valo raci\%C3\%B3ncondici\%C3\%B3nf\%C3\%ADsica.pdf

(14) Davó-Blanes MC, García de la Hera M, La Parra D. Educación para la salud en la escuela primaria: opinión del profesorado de la ciudad de Alicante. Gac Sanit. 2016; 30(1): 31-6.

(15) Agencia de Calidad de la Educación - Gobierno de Chile. Informe de Resultados Estudio Nacional de Educación Física 2014 [Internet]. Santiago de Chile: Agencia de
Calidad de la Educación; 2015. Disponible en: http:// archivos.agenciaeducacion.cl/Estudio_Nacional_Educacion_ Fisica_2014_8basico.pdf

(16) Delgado $P$, Caamaño F, Guzmán IP, Jerez D, Ramírez-Campillo R, Campos C, et al. Niveles de obesidad, glicemia en ayuno y condición física en escolares chilenos. Nutr Hosp. 2015; 31(6): 2445-50.

(17) Stewart A, Marfell-Jones M. International Standards for Anthropometric Assessment. ISAK, editor. Potchefstroom, Sudáfrica: International Society for the Advancement of Kinanthropometry; 2006.

(18) Unidad de Nutrición del Ministerio de Salud, Consejo Asesor en Nutrición, Grupo de Expertos. Norma Técnica De Evaluación Nutricional del Niño de 6 a 18 años. Año 2003. Rev Chil Nutr. 2004; 31(2): 128-37.

(19) Agencia de Calidad de la Educación - Gobierno de Chile. Informe de Resultados SIMCE Educación Física 8. Básico 2012 para Docentes y Directivos [Internet]. Santiago de Chile: Agencia de Calidad de la Educación; 2013. Disponible en: http://www. agenciaeducacion.cl/wp-content/uploads/2013/08/WEB_ IR_8_basico_Ed_Fisica_2012.pdf

(20) Mayörga-Vēga D, Mérino-Marban R, Rodríguez-Fernández E. Relación entre la capacidad cardiorrespiratoria y el rendimiento en los tests de condición física relacionada con la salud incluidos en la batería ALPHA en niños de 10-12 años. (Relationship between cardiorespiratory fitness and performance in the ALPHA health-related physical fitness test battery for 10-12 year-old children). Cult Cienc Deporte. 2013; 8(22): 41-7.

(21) Díez-Fernández A, Sánchez-López M, Mora-Rodríguez R, Notario-Pacheco B, Torrijos-Niño C, Martínez-Vizcaíno V. Obesity as a mediator of the influence of cardiorespiratory fitness on cardiometabolic risk: a mediation analysis. Diabetes Care. 2014; 37(3): 855-62.

(22) Druce Axley J, Werk LN. Relationship Between Abdominal Adiposity and Exercise Tolerance in Children With Obesity. Pediatr Phys Ther. 2016; 28(4): 386-91.

(23) Agencia de Calidad de la Educación - Gobierno de Chile. Informe de Resultados Estudio Nacional de Educación Física 2015 [Internet]. Santiago de Chile: Agencia de Calidad de la Educación; 2016. Disponible en: http://archivos. agenciaeducacion.cl/Informe_Nacional_EducacionFisica2015. pdf

(24) Khan S, Little J, Chen Y. Relationship Between Adiposity and Pulmonary Function in School-Aged Canadian Children. Pediatr Allergy Immunol Pulmonol. 2014; 27(3): 126-32.

(25) Caamaño F, Delgado P, Guzmán IP, Jerez D, Campos C, Osorio $A$. La malnutrición por exceso en niños-adolescentes y su impacto en el desarrollo de riesgo cardiometabólico y bajos niveles de rendimiento físico. Nutr Hosp. 2015; 32(6): 2576-83.

(26) Hernández C, Hernández D, Caniuqueo A, Castillo H, Fernandes da Silva S, Pavez-Adasme $G$, et al. Tablas de referencia para aspectos antropométricos y de condición física en estudiantes varones chilenos de 10 a 14 años. Nutr Hosp. 2016; 33(6): 1379-84.

(27) Pedraza DF, Silva FA, Melo NLS de, Araujo EMN, Sousa CP da C. Estado nutricional e hábitos alimentares de escolares de Campina Grande, Paraíba, Brasil. Ciênc Saúde Colet. 2017; 22(2): 469-77. 
(28) Ganie MA, Bhat GA, Wani IA, Rashid A, Zargar SA, Charoo $B A$, et al. Prevalence, risk factors and consequences of overweight and obesity among schoolchildren: a crosssectional study in Kashmir, India. ] Pediatr Endocrinol Metab. 2017; 30(2): 203-9.

(29) Jakšić M, Martinović M, Belojević G, Kavarić N, Ašanin B, Samardžić $M$, et al. Prevalence of and contributing factors to overweight and obesity among the schoolchildren of Podgorica, Montenegro. Srp Arh Celok Lek. 2017; 145(1-2): 20-5.

(30) Hernández-Cordero S, Cuevas-Nasu L, Morales-Ruán MC, Humarán IM-G, Ávila-Arcos MA, Rivera-Dommarco JA. Overweight and obesity in Mexican children and adolescents during the last 25 years. Nutr Diabetes. 2017; 7(3): e247.

(31) Cristi-Montero C, Celis-Morales C, Ramírez-Campillo R, AguilarFarías N, Álvarez C, Rodríguez-Rodríguez F. iSedentarismo e inactividad física no son lo mismo!: una actualización de conceptos orientada a la prescripción del ejercicio físico para la salud. Rev Med Chil. 2015; 143(8): 1089-90.

(32) Vásquez F, Díaz E, Lera L, Meza J, Salas I, Rojas P, et al. Efecto residual del ejercicio de fuerza muscular en la prevención secundaria de la obesidad infantil. Nutr Hosp. 2013; 28(2): 333-9.

(33) Ranson R, Stratton G, Taylor SR. Digit ratio (2D:4D) and physical fitness (Eurofit test battery) in school children. Early Hum Dev. 2015; 91(5): 327-31.

(34) Cárcamo-Oyarzún J, Sanhueza $S$, Agüero $H$, Cumilef $P$, González Y, Hernández D, et al. Valores de Referencia para Fuerza Muscular y Flexibilidad en Escolares de 13 y 14 Años de la Región de Aysén y sus diferencias según género y tipo de
Establecimiento Educacional. Rev Cienc Activ Física IND. 2014; 9: 45-56.

(35) Sacchetti R, Ceciliani A, Garulli A, Masotti A, Poletti G, Beltrami $P$, et al. Physical fitness of primary school children in relation to overweight prevalence and physical activity habits. J Sports Sci. 2012; 30(7): 633-40.

(36) Hernández C, Fernandes da Silva S, Ibarra J, Hernández $D$, Caniuqueo A, Esparza $E$, et al. Nivel de condición física orientada a la salud en estudiantes varones de 10 a 14 años del colegio Dario Salas, Chillan. Mot Hum. 2015; 16(1): 33-40.

(37) Kodama S, Saito K, Tanaka S, Maki M, Yachi Y, Asumi M, et al. Cardiorespiratory fitness as a quantitative predictor of allcause mortality and cardiovascular events in healthy men and women: a meta-analysis. JAMA. 2009; 301(19): 2024-35.

(38) González-Gross M, Meléndez A. Sedentarism, active lifestyle and sport: Impact on health and obesity prevention. Nutr Hosp. 2013; 28 Suppl 5: 89-98.

(39) Dinsa GD, Goryakin Y, Fumagalli E, Suhrcke M. Obesity and socioeconomic status in developing countries: a systematic review. Obes Rev. 2012; 13(11): 1067-79.

(40) Villamor E, Finan CC, Ramirez-Zea M, Roman AV, Nine Mesoamerican Countries Metabolic Syndrome Study (NiMeCoMeS) Group. Prevalence and sociodemographic correlates of metabolic syndrome in school-aged children and their parents in nine Mesoamerican countries. Public Health Nutr. 2017; 20(2): 255-65.

(41) Garcia-Rubio J, Lopez-Legarrea P, Gomez-Campos R, CossioBolaños M, Merellano-Navarro E, Olivares PR. Ratio CinturaEstatura y riesgo de Síndrome Metabólico en adolescentes chilenos. Nutr Hosp. 2015; 31(4): 1589-96. 